\title{
A CURIOUS SYNDROME, WITH SIGNS SUGGESTING CERVICAL ARTERIO-VENOUS FISTULA, AND THE PULSES OF NECK AND ARMS LOST
}

\author{
BY \\ THOMAS LEWIS AND JOAN STOKES \\ From the Department of Clinical Research, University College Hospital Medical School \\ Received March 24, 1942
}

A married woman of 33 years was admitted to hospital on November 25, 1941 , complaining of attacks of faintness. She is said to have been rickety as a child and was not allowed to swim at school. After growing up she attended hospital for deafness of her left ear. A roctor ?xamining her in her twenty-sixth year found no pulse at the wrist.

She states that her fainting attacks have been present for about six years and that recently they have become more frequent and now occur almost daily. In the attack her vision becomes dim, she feels weak and would fall without support, but she does not believe she loses consciousness. Her tongue has never been bitten nor her water passed in the attacks. They occur while she is up and about, and also when she is in bed, but especially when she sits up in bed. They are usually followed by headache. She has dropped things from her hand while carrying them, and has collapsed and fallen in the street, but has never hurt herself.

Headaches have been frequent recently; they are severe and felt over the top of the head. She lost the sight of her right eye two years ago in one of her attacks. When scrubbing or doing other hard work she has to stop from time to time owing to aching pain in her arm. Her fingers sometimes become numb and cold without apparent reason.

She has had no children; a pregnancy nine months ago was terminated.

Condition.-She is a small, thin woman (weight, 6 st. 5 oz.). No abnormalities are found in her nervous system, except nerve deafness in the right ear, and a primary optic atrophy in the fundus of the right eye; the vessels of the fundus are normal and there is no exudate. The face is warm and pale and the hands are usually warm and of good colour. Her temperature is usually subnormal; the heart rate varies between 75 and 100 a minute while she lies in bed. Her respirations are 20-24 a minute. The Wassermann and Kahn reactions are repeatedly negative. The blood count is normal. X-rays show no defect in the optic foramina, but the clinoid processes (anterior and posterior) on the right side are eroded.

Heart.-The venous pressure and the venous pulse are normal. The $\mathbf{F}$ 
heart is not enlarged to percussion; the impulse is in the fifth space, $10 \mathrm{~cm}$. from the midline. The sounds are cleariy struck and the action regular. The $\mathrm{X}$-ray silhouette, including the arch of the aorta, is normal in outline, but the aortic shadow and that of the basal vessels is rather dense. There is no tracheal tug. The electrocardiogram is normal in its initial phases in all leads.

Arteries and blood pressure.-No pulse can be felt in any artery of either arm, head, or neck. In the neck the pulseless carotid arteries can be felt as unusually firm smooth round cords throughout their length. The innominate vessel can be felt crossing the trachea in the episternal notch; it pulsates palpably though not strongly and is continued into the pulseless carotid vessel. The Pachon oscillometer gives no movement from the upper arms, but the brachial arteries are easily felt as unusually firm round cords throughout their length. The abdominal aorta pulsates freely, the femoral pulses are powerful, and the pulses of the feet are easily palpable, but not waterhammer in quality.

The blood pressure in the popliteal artery, read on a number of occasions, is 210-230 systolic (readings of two years ago were similar) and 130 diastolic. In the arm, blood pressure is obtained by holding the arm aloft for a moment and occluding all circulation by means of the pressure cuff; and then, after an interval of three minutes, allowing the pressure to fall in steps until the hand flushes. Sharp readings can be obtained in this way, showing the mean pressure to be 45 to $55 \mathrm{~mm}$. It can also be shown that the brachial arteries are carrying blood; for if, when the circulation to the limb has been occluded, the brachial artery is firmly gripped and the pressure in the cuff then deflated, reactive hyperæmia is delayed until the brachial artery is released, say fifteen or twenty seconds later, while the hyperæmia occurs in two or three seconds if the brachial artery has not been held. Similarly it can be shown that the subclavian artery is carrying blood; for if the artery is held below the clavicle the colour begins to return only slowly to the hand in five seconds and is full at ten seconds; whereas it returns almost at once if the subclavian artery is free. Compression of the left carotid artery, but not the right, is usually followed by an attack of faintness and pallor, with flushing of the face on release.

Just above the right sternoclavicular joint a systolic thrill and a continuous murmur, louder in systole, are conspicuous. The murmur is heard maximally at the black circle of the diagram, but extends over the whole sternal, supraclavicular, and supracapular regions (cross-hatched area). The systolic thrill is synchronous with the innominate impulse. No unusual pulsation is to be felt in this region. The veins of the neck are inconspicuous and undistended while the patient lies recumbent.

Anastomotic arteries.-A systolic murmur is heard over large areas of the chest and abdominal wall (the hatched areas of the chart). The areas of the systolic murmur are similar to those heard over the anastomotic vessels in coarctation of the aorta, as previously reported (Lewis, 1933). In the present case arterial pulsations are also to be felt in several places on the abdominal wall; one conspicuous pulsation is present to the right of the navel and the systolic murmur is loud here. A faint pulse has been felt at one or two points 
on the back of the chest too, and a systolic murmur is audible at the angle of the right scapula. It seems clear that the chief anastomoses unite arteries of the neck and sternal regions, and with especial freedom on the right side, through the epigastric and internal mammary arteries. An indication of the direction of flow in the main anastomotic arteries is obtained by timing the

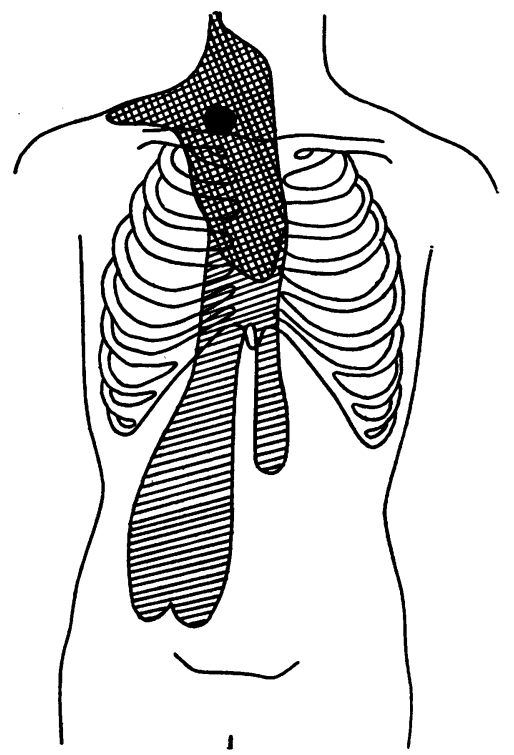

Fig. 1.-Case 1. The area where the systolic murmur could be heard is hatched and the area of the continuous murmur is cross-hatched. The maximal point of thrill is marked by the black circle above the clavicle.

pulsations graphically. The innominate pulse precedes the femoral at Poupart's ligament by about $0.1 \mathrm{sec}$; the pulsations of an anastomotic artery near the navel precedes that of the femoral by a little less than this value. Thus the order is innominate, abdominal wall, femoral; closure of the common femoral artery is without effect on pulsations in the abdominal wall. The third and fourth ribs present a few small but unusual crenations of their lower borders.

Renal functions.-The urine is of normal quantity and specific gravity and contains no abnormal constituent. The urea concentration test gives a value as high as 3.09 ; the urea clearance is 70 per cent of normal, and the blood urea $30 \mathrm{mg}$. per 100 c.c.

The attacks.-A number of the attacks have been witnessed by her husband, by nursing staff, and by patients in adjoining beds. These attacks are brief, lasting usually ten to twenty seconds, and once about two minutes. Pallor with subsequent cyanosis and noisy breathing are usually noticed. Rigidity, with head retraction, is usual, and unconsciousness not infrequent. Sometimes in the attacks movements of rotation occur in the forearms. In one attack the heart accelerated to 144 a minute, and the patient complained subsequently that her whole right arm had become paralysed for a few moments. Attacks have been provoked by sitting up or kneeling up in bed and leaning over her locker; but the patient always has time to resume the supine position. 


\section{Discussion}

The patient described presented signs of a very unusual vascular anomaly. No pulsation could be found in either of the carotid or subclavian arteries; the mean blood pressure in the arms was only $50 \mathrm{~mm}$.; and the arms readily developed the ache of muscular ischæmia when used. The patient suffered from frequent attacks of faintness or transient loss of consciousness, and from a unilateral optic atrophy of a few years standing. These evidences all point to a grossly deficient circulation to the head and neck and to the two upper limbs.

The establishment of a great difference between the pressures in upper and lower parts of the body in such a case as this has a clear physiological basis; the low pressure in the upper parts, and presumably in the carotid regions especially, will call forth persistent vasoconstriction, and this will raise the pressure in the lower parts. It is here to be noted that no direct explanation other than the physiological could be found in our patient to explain the raised blood pressure. High pressure in the legs has been recorded in cases of aneurysm with pulseless upper parts by Maurer (1939), Kampmeier and Neumann (1930), and Cohen and Davie (1933). Thus our case in following physiological expectation appears to conform to a rule.*

The aorta itself was unchanged in form and size, its ascending and transverse parts being clearly visible in the X-ray, and the pulse being transmitted without undue delay to the femoral arteries. A pulsation, promptly delivered from the heart, was readily felt in an artery that was regarded from its size and position as the innominate, at the root of the neck; this vessel could be traced upwards into the firm non-pulsating cord of the right carotid. The deficient circulation to the upper parts of the body was manifestly due to an impervious condition, relative or absolute, of subclavian and carotid arteries on both sides.

In most of the instances of lack of pulsation in both carotids and subclavians previously recorded, the patients have been proved to be syphilitic, and those coming to post-mortem examination have been cases of aneurysm of the aorta, the obstruction being due to clot extending into and plugging the mouths of the arteries arising from the arch of the aorta, or to closure of these mouths by sclerosis. Such an explanation is not open to us in the case of a young woman, giving repeatedly negative Wassermann and Kahn reactions,

* Such conformity re-emphasizes the curious blood pressure picture in cases of coarctation of the aorta. The condition as a whole might be likened to one of inverted coarctation of the aorta in its effects on the general pressures. Giffin and Dill have already drawn this analogy in the description of a not dissimilar case.

In 1933 the blood pressure readings from previously recorded cases of coarctation of the aorta were collected for the first time, and to these were added those from eight newly recorded cases (Lewis, 1933). From this material it was possible to conclude that high blood pressure in the upper parts of the body is the rule in these cases, while low pressure prevails in the lower parts. It could not be overlooked that the high pressure in the upper half of the body might be due to a mechanism set in motion by the need of the lower part of the body, but, when examined, the nature of this mechanism remained, and still remains, obscure. Goldblatt, Kahn, and Hanzal (1939) have since shown that clamping the abdominal aorta just above the renal arteries causes a delayed hypertension to develop, as a result of renal ischæmia. Possibly a similar mechanism applies to coarctation, though the freedom of anastomoses and the infrequency of renal insufficiency in these cases is to be noted. 
and presenting under X-ray no trace of aortic dilatation or distortion. Giffin (1939) in describing a case very similar to the present one, a woman of 19 years, was for similar reasons precluded from assigning it to syphilis; and consequently in the discussion of his case, a congenital anomaly of arteries was suggested as the possible basis of the lesion. It is but a suggestion, for no such anomaly is known in which all of the three usual vessels, innominate, subclavian, and carotid, fail; or which would account for loss of pulses in head, neck, and arms. We may place the argument on more general lines. When a usual artery, such as those named, fails to arise from its accustomed parent stem, it arises from some other source. The congenital anomalies of arteries are not suppressions of arterial supply, they are merely unusual arrangements of supply. In thinking of a developmental failure of arterial supply, it is to be remembered that the first arrangement of main arterial paths is determined at an early stage of uterine life, at a time when the limbs have still to make the great bulk of their growth. That adequate arterial development should fail while the tissues and structures of the corresponding territory continue to develop normally from this early stage is inconceivable. The evidence from our patient is of a gross defect of circulation to the head and limbs. To account for this state an anomaly of development would be, by itself, an inadequate explanation.*

It would seem necessary to adopt an explanation embracing an acquired lesion. The nature of the acquired lesion remains obscure; a chief clue to it being the remarkable cord-like condition of the main arteries in the neck and arms, already described. Extensive thrombosis might be expected to have produced at the time sudden and emphatic cerebral symptoms; it might also be expected to have rendered the vessels concerned quite impervious; yet as we have seen the brachial arteries and probably the left carotid, though greatly thickened, were not impervious. A dissecting aneurysm spreading to the basal vessels is a possible explanation, but there is no definite support for the suggestion.

In searching for an interpretation of this curious case there is another feature which stands out and calls for explanation, namely, the continuous murmur and thrill at the base of the neck. It is such a murmur and thrill as is known to occur only where a main artery is in communication with another vessel under much lower tension. Simple anastomoses are not known to give continuous murmurs. Although an arteriovenous fistula is the most probable explanation, yet it may be objected that increased pressure in the veins of the neck, and a steep-rising pulse in the legs, would be expected in association; neither of these phenomena occurred. Such a fistula can by no means be excluded, however, since it may have been deeply seated, as in the bones of the vertebræ or skull; no evidence in support could be found in X-ray pictures of the neck, though the sella turcica was seen to be malformed, the clinoid

\footnotetext{
* Coarctation of the aorta cannot legitimately be cited in the contrary sense, for this defect is not supposed to occur by failure of development, but by some form of interference, at or about the time of birth, causing the already open aorta to close down in the region of insertion of the ductus arteriosus.
} 
processes being small and obscure. No murmur could be heard over the head. The anastomosing vessels in the case are more consistent with the idea of an arterio-venous fistula than with that of a purely obstructive lesion necessitating collateral supply of the head and arms from the lower parts of the body. As has been seen, the collateral channels did not lead upwards through epigastric and internal mammary from femoral to supraclavicular region.

A point upon which considerable emphasis is to be laid is that Giffin's case, so like ours in other respects, also presented a continuous thrill in a similar situation. So did a patient whom we saw many years ago. This was a young woman, the pulse in whose neck was absent or almost imperceptible. The Wassermann reaction was negative, but the aorta was definitely dilated. This case is of special interest in that the blindness was known to have resulted from a recent obstruction of the central artery of the retina. The notes of this case, imperfect though they are, and an abstract of Giffin's case are appended. Here then are three cases of very similar type, and all presenting a similar murmur. The fact suggests that we are dealing with a pathological entity hitherto unrecognized and still awaiting post-mortem dissection before its precise form can be understood.

\section{ApPENDEd Notes of ANOTHER CASE}

Case 2. A housewife of 45 years was admitted to hospital in the autumn of 1932, complaining of attacks of faintness, and blindness of the right eye. She also suffered at this time from bronchitis. There was a history of rheumatic fever at 8 years and of white leg and varicose ulcers following pregnancy. She was the mother of seven children, five alive and well. One child came prematurely and died, and one died at fourteen months from rickets and pneumonia.

In March 1931, when in hospital for pregnancy, it was found that pulsation in her radial and carotid arteries was much diminished; this was the first record of any abnormality of her vascular system. The fainting attacks began during pregnancy in 1930 and occurred about once in five weeks; they were usually provoked by sitting up in bed in the morning and were also brought on by stooping. They comprised mistiness of vision, dizziness, and weakness in the left arm, followed by numbness and tingling and often by severe headache. Consciousness was not lost. The blindness came suddenly during an attack of " influenza."

Condition.-She was a healthy-looking and well-nourished woman (weight, 11 st. $5 \mathrm{lb}$.). Her colour was good and her hands and face warm. Her temperature was usually subnormal and her heart rate varied while she was in bed from 68-84 a minute. There were no abnormal signs in her central nervous system and the loss of vision in the right eye was stated by $\mathrm{Mr}$. $\mathrm{H}$. Neame to arise out of recent obstruction of the retinal artery. The retina was pale and œdematous, especially at the macula, which presented a bright red spot; the arteries were very thin and of irregular lumen, the veins smaller than normal; 
the œdema subsided subsequently. Her Wasserman reaction was found negative on two occasions.

Heart.-Venous pulsation and pressure in the neck were normal, and the heart was not enlarged. Some retrosternal dulness and pulsation could be seen in the third right space during full expiration. There was no pulsation of the sternum and no tracheal tug. X-ray of the chest confirmed that the

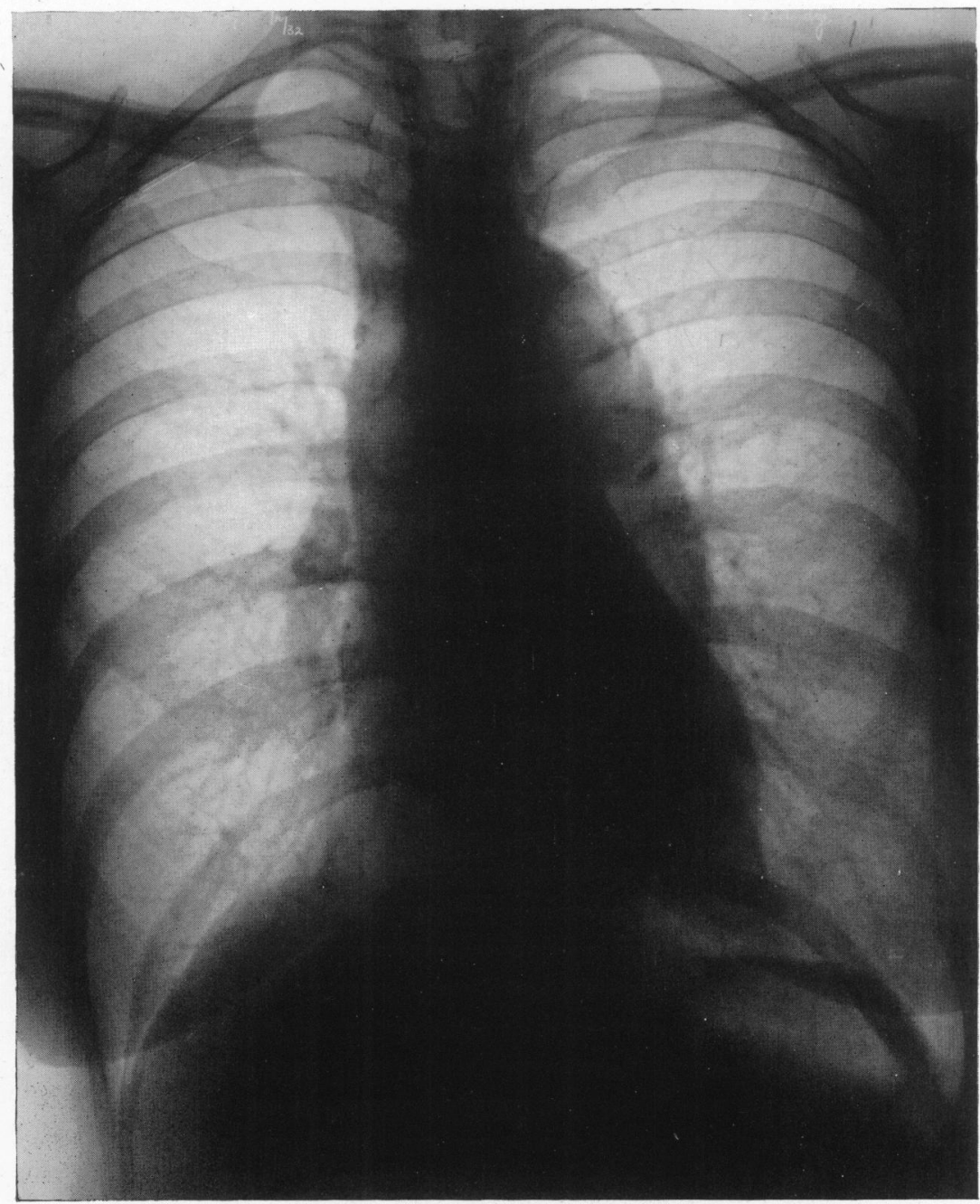

Fig. 2.-Case 2. Antero-posterior radiogram of the chest, taken on October 12, 1932.

heart was normal in size and showed a diffuse dilatation of the aortic arch and a shadow running from the arch of the aorta, parallel to the right border of the sternum to the region of the sterno-clavicular joint, presumably a dilated innominate vessel (see Fig. 2). There was no notching of the ribs such as is seen in cases of coarctation of the aorta. The electrocardiogram was normal. 
Arteries and blood pressure.-In the right radial artery only a flicker of pulsation could be felt. The right brachial and carotid, and the left radial, brachial, and carotid arteries were not felt to pulsate.

Both femoral arteries presented a strong but rather slow-rising pulse, and pulsation in the dorsalis pedis arteries was easily felt. A murmur was heard maximally at the base of the neck in the angle between the right sterno-mastoid muscle and the clavicle. It was loudest in systole and prolonged almost or quite into diastole, resembling to several observers the murmur of a patent ductus arteriosus. It was conducted up the neck as far as the angle of the jaw on the right, and a little way up on the left, down the right border of the sternum as far as the fourth space, and over the manubrium. A thrill, localized at the base of the neck, accompanied it, but there was no pulsation in this region. An unexplained systolic murmur was also heard below the angle of the left scapula; it is not clear that any other signs of anastomoses were sought. The Pachon oscillometer reading showed small pulsations from both arms. The maximal pulsation occurred at $60 \mathrm{~mm}$. in the arm and at $100 \mathrm{~mm}$. in the leg. The systolic blood pressure in the legs varied between $130-150 \mathrm{~mm}$.

When the patient was previously admitted in March 1931, her signs were a little different. At that time very slight pulsation was felt in both radial and carotid arteries and the blood pressure in the arms was recorded at 70 to $80 \mathrm{~mm}$. Dilatation of the aorta, as revealed to X-ray, was then not quite so great. Vision at this time was good and the fundus oculi was normal on both sides.

The urine contained neither blood nor albumin; renal function tests were not done.

\section{Abstract of Dr. Giffin's CaSE}

Case 3. A woman, aged 19, came to hospital in December 1938 because of headaches and failing vision.

In the spring of 1938 she began to have attacks of blurred vision lasting for one or two minutes. These episodes increased in frequency until August 1938, when she was confined to bed. Since then she had returned to limited activities and could read except during daily transitory attacks. Her sight was poorest on rising and improved during the day. She also complained of bilateral frontal headaches for several years and occipital headaches intermittently for nine months. Vomiting had occurred occasionally. The family history was not significant. She was normal at birth but was weak and under-developed in childhood. Later, owing to weakness, she was never able to do housework. Her hands and jaws fatigued easily and she had always been pale.

Condition.-The patient was mentally retarded and physically underdeveloped. Her central nervous system was normal. Her face and mucous membranes were pale.

Pulsation could not be felt in the left radial, brachial, subclavian, or carotid arteries. It was also absent in the right radial, brachial, and subclavian arteries, but in the right carotid artery diminished pulsation with a slight thrill was felt at the level of the cricoid cartilage, and a loud continuous bruit was heard 
at this point. Photographic record of the murmur showed that it was continuous throughout the cardiac cycle, and it was thought to indicate the presence of arterio-venous fistula. Pressure medial to the right carotid artery, however, stopped the murmur without significant alteration in the heart rate. The pulsation of the abdominal aorta and its branches was somewhat increased. In the legs oscillometer excursions were normal and the blood pressures varied from 175-190 systolic and 90-110 diastolic. Skin temperatures of the fingers of both hands were consistently lower than those of the toes.

The heart was normal in size. A systolic murmur maximum in the second left interspace was heard and the pulmonary second sound was accentuated. X-ray revealed a relatively small heart, the absence of an aortic knob, and a prominent pulmonary conus. There were no cervical ribs, no rib notches, and no bony erosion in the cervical region. Radiograms of the skull were normal. The electrocardiogram revealed nothing diagnostic and the Wassermann reaction was negative.

Examination of the ocular fields showed an "upper altitudinal" scotoma on the left. Vision was $6 / 30$ in the left eye and $6 / 10$ in the right eye. There was no papillœdema. Fragmentary flow of blood was seen in the retinal veins and also several ischæmic areas were found. Retinal pressures, measured by an indirect method were found to be less than $20 \mathrm{~mm}$. of mercury on the right and were unobtainable on the left.

\section{SUMMARY}

A patient is described in whom the pulses of the neck and arms had been lost, and who presented signs suggesting an arterio-venous fistula at the root of the neck. The defective blood supply to the upper parts of the body was responsible directly or indirectly for a gross defect of vision, for frequent fainting attacks and headaches, and for pain in the right arm during work. The blood pressure in the patient's legs was raised.

Another case of a very similar kind is described, and a third recorded by Giffin is compared with them.

The three cases show so much in common as to suggest a pathological entity hitherto unrecognized and still awaiting dissection before its precise form can be understood.

\section{REFERENCES}

Cohen, H., and Davie, T. B. (1933). Lancet, 1, 352.

Giffin, H. M. (1939). Proc. Staff Meet. Mayo Clinic, 14, 561.

Goldblatt, H., Kahn, J. R., and Hanzal, R. F. (1939). J. exper. Med., 69, 649.

Kampmeier, R. H., and Neumann, J. F. (1930). Arch. intern. Med., 45, 513.

Lewis, T. (1933). Heart, 16, 205.

Maurer, E. (1939). Amer. Heart J., 17, 716. 